\title{
The Nature and Extent of COVID-19 Vaccination Hesitancy in Healthcare Workers
}

\author{
Nirbachita Biswas ${ }^{1} \cdot$ Toheeb Mustapha $^{1}$. Jagdish Khubchandani ${ }^{1}$. James H. Price ${ }^{2}$ \\ Accepted: 23 March 2021 / Published online: 20 April 2021 \\ (c) The Author(s), under exclusive licence to Springer Science+Business Media, LLC, part of Springer Nature 2021
}

\begin{abstract}
COVID-19 vaccines were approved in late 2020 and early 2021 for public use in countries across the world. Several studies have now highlighted COVID-19 vaccination hesitancy in the general public. However, little is known about the nature and extent of COVID-19 vaccination hesitancy in healthcare workers worldwide. Thus, the purpose of this study was to conduct a comprehensive worldwide assessment of published evidence on COVID-19 vaccine hesitancy among healthcare workers. A scoping review method was adopted to include a final pool of 35 studies in this review with study sample size ranges from $\mathrm{n}=123$ to 16,158 (average $=2185$ participants per study). The prevalence of COVID-19 vaccination hesitancy worldwide in healthcare workers ranged from 4.3 to $72 \%$ (average $=22.51 \%$ across all studies with 76,471 participants). The majority of the studies found concerns about vaccine safety, efficacy, and potential side effects as top reasons for COVID-19 vaccination hesitancy in healthcare workers. The majority of the studies also found that individuals who were males, of older age, and doctoral degree holders (i.e., physicians) were more likely to accept COVID-19 vaccines. Factors such as the higher perceived risk of getting infected with COVID-19, direct care for patients, and history of influenza vaccination were also found to increase COVID-19 vaccination uptake probability. Given the high prevalence of COVID-19 vaccine hesitancy in healthcare workers, communication and education strategies along with mandates for clinical workers should be considered to increase COVID-19 vaccination uptake in these individuals. Healthcare workers have a key role in reducing the burden of the pandemic, role modeling for preventive behaviors, and also, helping vaccinate others.
\end{abstract}

Keywords COVID-19 $\cdot$ Vaccine $\cdot$ Healthcare worker $\cdot$ Health professionals $\cdot$ Vaccination

\section{Background}

COVID-19 vaccines were approved for use in the general population in late 2020 and early 2021 across different countries. COVID-19 vaccination hesitancy rates in the general population have now been explored across the world and are fairly well established [1-4]. For example, in a recent systematic review, the highest rates for COVID-19 vaccine acceptance in the general population were reported in Ecuador, Malaysia, Indonesia, and China ( $>90 \%$ for all countries). In contrast, the lowest rates were reported for Kuwait, Jordan, Italy, Russia, Poland, United States, and

Jagdish Khubchandani

jagdish@nmsu.edu

1 Department of Public Health, New Mexico State University, Las Cruces, NM 88003, USA

2 School of Population Health, University of Toledo, Toledo, OH 43606, USA
France ( $<60 \%$ for all countries) [4]. In these studies, from the general population, it has been shown that COVID-19 vaccination hesitancy rates differ worldwide by perceived susceptibility to and severity of COVID-19 and several sociodemographic characteristics such as sex, age, education, income, and occupation [2-5].

Recent reports suggest that many healthcare workers (HCWs) are also hesitant about or are delaying getting the COVID-19 vaccine [6-10]. Some reports estimate that the rates of COVID-19 vaccination hesitancy in HCWs may be similar to rates in the general population. For example, a December 2020 Kaiser Family Foundation poll found that $29 \%$ of the HCWs were reluctant to get COVID-19 vaccines as opposed to $27 \%$ of the individuals in the general population [8]. In contrast, in an early 2021 assessment of skilled nursing facilities across the United States, more than threefourths $(77.8 \%)$ of the residents of these facilities compared to a little more than a third $(37.5 \%)$ of the staff in these facilities received at least one dose of the COVID-19 vaccine 
[9]. These estimates are of concern even though HCWs were designated as priority groups for COVID-19 vaccination across the world [8-10]. Given the scattered scientific evidence and a plethora of media reports, it remains to be known to what extent and why are HCWs hesitant towards obtaining COVID-19 vaccination. Thus, the purpose of this study was to review all published scientific evidence on the extent and reasons for COVID-19 vaccination hesitancy in HCWs. Also, we explored the enabling factors for COVID19 vaccination across the world to assimilate similarities and differences in HCWs across the world as it relates to COVID-19 vaccination acceptance or willingness.

\section{Methods}

We conducted a scoping review to assimilate scientific evidence on COVID-19 vaccine hesitancy in HCWs. A comprehensive search of the scientific literature was conducted by three independent investigators (NB, TM, JK) on PubMed, EBSCO Host, pre-print servers (e.g. medRxiv) and Google Scholar. A Boolean search strategy was employed using the following keywords: "vaccine", "COVID-19", "hesitancy", "refusal", "vaccination", "coronavirus", "nurse", "doctor", "healthcare", "worker", "health", "professional". The order of keywords was changed in repeated searches across databases to extract the final pool of relevant studies. Articles that were included in the final review were screened carefully for suitability and to identify other articles that pertained to the study topic. Additional hand searches were conducted to include articles that cited the initially selected pool of articles. COVID-19 vaccination hesitancy rates data were extracted from studies if participants were "unlikely", "refused", "declined", or "disagreed" with taking a COVID-19 vaccine. Participant responses such as "unsure" and "undecided" were not counted as vaccine hesitant for assessment of COVID-19 vaccination rates among the studies included in this review. Studies finally included in the review were those that were published in the English language, between February 2020- February 2021, and included healthcare workers as sole study participants (e.g., doctors, nurses, allied health professionals, and others) or HCWs who were part of a survey from the public.

\section{Results}

The final pool of studies comprised 35 studies ( 9 from the USA; 4 from France; 3 from Saudi Arabia; 3 from Hong Kong/ China; 2 from Congo; 2 from Malta; 2 from Greece, 2 from Italy, 2 from Canada, and 1 each from other countries as shown in Table 1) [11-45]. The sample size of the studies ranged from 123 to 16,158 participating HCWs (average study sample $=2185$ per study and a total of 76,741 participants across all studies). The prevalence of COVID-19 vaccination hesitancy worldwide in HCWs ranged from 4.3 to $72 \%$ (average rate of $22.51 \%$ hesitant individuals across studies). The type of HCWs in studies included for this review differed by age, gender, race, education, income, marital status and family structure, profession, and roles in the healthcare setting (e.g., direct patient contact versus others) [11-45]

In the studies where reasons for COVID-19 vaccination hesitancy were assessed, the vast majority ( $>75 \%)$ of the studies found concerns about safety, efficacy, and side effects as the top three major reasons for COVID-19 vaccination hesitancy among HCWs. Other reasons for COVID-19 vaccination hesitancy were: insufficient knowledge about the vaccines, belief that COVID-19 does not exist or is not a serious disease, speedy development of vaccines, politics surrounding vaccine development process, misinformation from social media, previous COVID-19 infection or health conditions, and mistrust in authorities, health experts, and pharmaceutical companies.

Concerning factors associated with lower COVID-19 vaccination hesitancy and higher willingness for COVID19 vaccines, the male gender was found to be an enabling factor in the majority of the studies $(25 / 35=71.4 \%)$. Older age or having a doctorate or postgraduate education were associated with higher COVID-19 vaccine acceptance rates in more than half of the studies $(23 / 35=65.7 \%)$. Compliance with vaccines, confidence in vaccines, or history of influenza vaccination was found to be a predictor of COVID-19 vaccine acceptance in a little more than half of the studies $(18 / 35=51.4 \%)$. Similarly, direct patient contact/ caring for COVID patients or higher perceived risk and fear of being infected with COVID-19 were associated with lower COVID-19 vaccination hesitancy in more than half of the studies $(20 / 35=57.1 \%)$. Other factors associated with higher COVID-19 vaccination acceptance were: White or Asian race, higher income/education, medical risk, and chronic disease history, not being infected with COVID-19 in the past, knowledge about COVID-19 infection and disease, working in non-rural areas, and beliefs that vaccines may protect friends, family, and community members.

\section{Discussion}

The findings of this review indicate that $22.51 \%$ out of 76,471 HCWs worldwide reported COVID-19 vaccination hesitancy. The public would assume that HCWs would have no hesitancy to take the COVID-19 vaccine, given the nature of the profession of HCWs. A recent survey from the general public in the United States $(n=1055)$ found that $32 \%$ of the participants would not or probably not get the COVID-19 


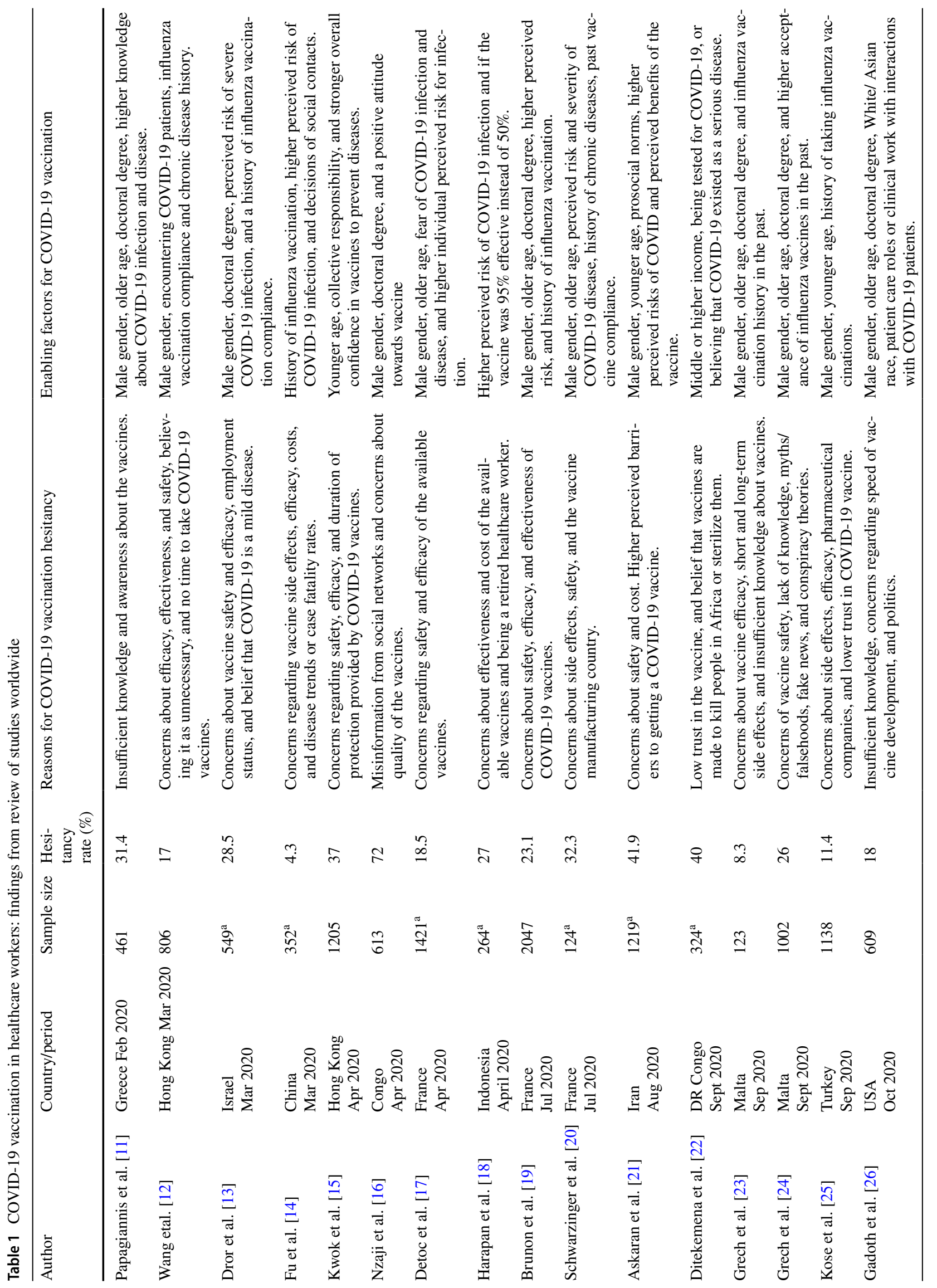




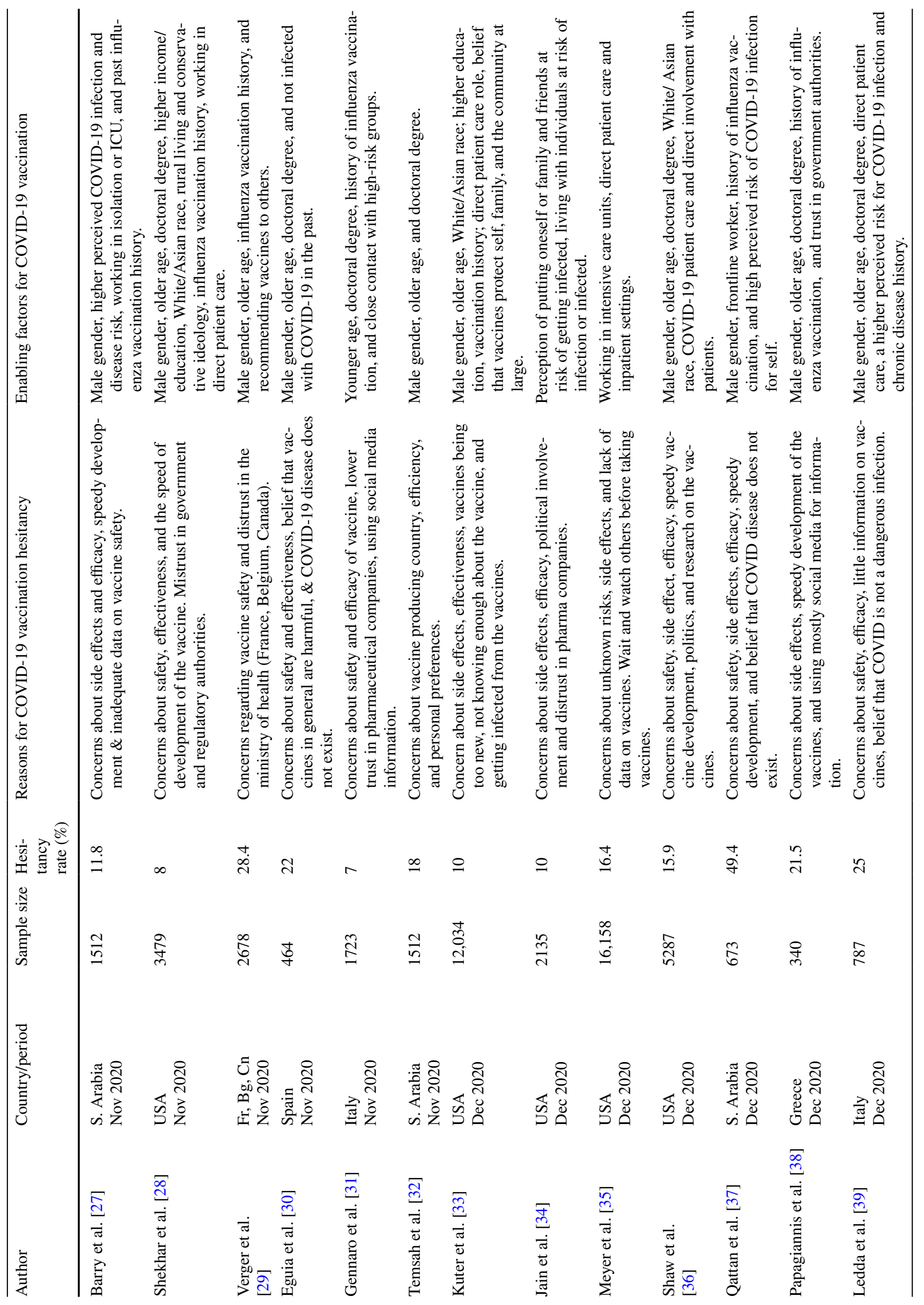




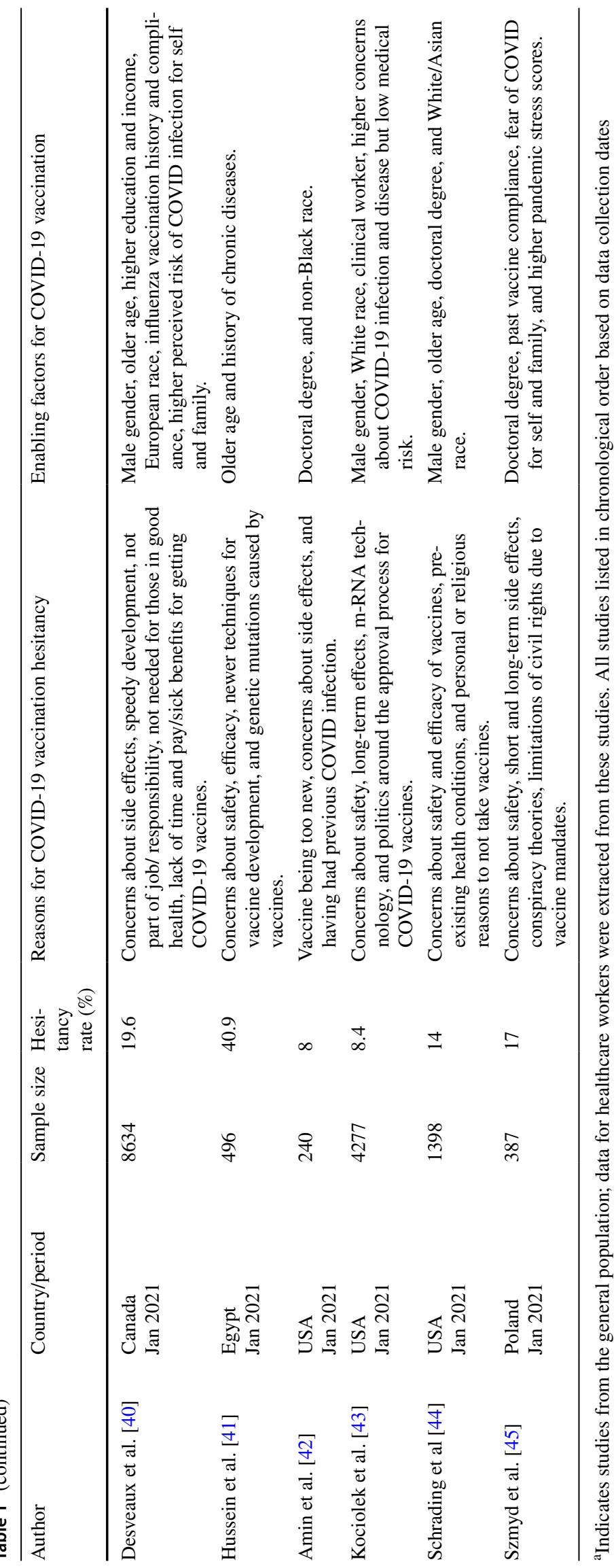


vaccine [46]. Their main concerns with the vaccine were potential side effects (60\%), safety (48\%), how well it works $(30 \%)$, and not trusting the government $(22 \%)$. These reasons for not getting vaccinated were very similar to the reasons found for HCWs in this review: concerns about safety, efficacy, and side effects of the COVID-19 vaccines. HCWs' willingness to be COVID-19 vaccinated serves an important role model function for the public. Studies have found that the public trusts physicians, hospitals, researchers, and health authorities to do the right thing regarding COVID-19. Also, studies from the general public on COVID-19 vaccination hesitancy have found that recommendations by healthcare providers are associated with lower odds for COVID-19 vaccination hesitancy [14, 29, 40, 47]. However, if HCWs continue to remain hesitant towards COVID-19 vaccines, it is unlikely that they would recommend these vaccines to the general public and ensure mass vaccinations with the available COVID-19 vaccines.

Several other sociodemographic characteristics that influence COVID-19 vaccination acceptance in the general public are also associated with COVID-19 vaccination acceptance or refusal in HCWs. For example, male HCWs were more likely to accept vaccines in this review. In the general public, it has been found that females are less likely to accept a COVID-19 vaccine and it has been postulated that this could be due to concerns about side effects such as infertility, serious side effects making them unable to take care of families, or greater susceptibility to myths and misinformation from media $[3,6,13,24,40,45]$. A few studies on HCWs in this review also found higher vaccine acceptance in Whites and Asians. In the general public, COVID-19 vaccination hesitancy is well established among racial and ethnic minorities and the reasons could be similar irrespective of occupational groups (e.g., medical mistrust, history of discrimination and mistreatment in research, etc.) $[3,6,9,28]$. Similar to findings in the general public, age was also a determinant for COVID-19 vaccination acceptance in HCWs; older HCWs were more willing to accept COVID-19 vaccines. This could be because older individuals may have higher education, greater experience in healthcare settings, greater perceived vulnerability to COVID-19 infection, or higher overall medical and health risk profiles $[3,5-8,40]$. Lower education is another factor associated with COVID-19 vaccination hesitancy in both HCWs and the general public. HCWs with lower education may not read the recent research, may have lower awareness or perceived risk of illness from COVID19 , lower past compliance with vaccinations, or may have a greater tendency to believe in community myths (e.g., conspiracy theories, injection of microchips, genetic mutation or infection from the vaccine, etc.) [6-12].

There is a lot of confluence for the aforementioned sociodemographic characteristics outlined in our review that associate with higher COVID-19 vaccination in HCWs.
A significant proportion of HCWs in the studies reviewed were females, with lower education and income, lower perceived risk of illness and younger, and belong to racial/ minority populations. Frequently, these populations are overrepresented in the healthcare workforce as frontline, essential, or direct care workers [6-10]. Unfortunately, these are also the populations that have been most affected by the COVID-19 pandemic with a higher number of cases and deaths related to COVID-19 [3, 6, 9, 10]. Interdisciplinary and multipronged strategies must be utilized to increase COVID-19 vaccine uptake in these populations (e.g., incentives to be vaccinated, role models and community leaders encouraging vaccinations, educational interventions, hospital-based protocols and mandates, prioritizing vaccination for these groups and making vaccine access easy, giving time off or sick leave benefits to enable vaccination etc.) $[6,7,9,10,47,48]$.

Unlike the general public, HCWs have an obligation to "do no harm" to patients and a "duty to care" for patients who seek their help [48]. HCWs regularly work with vulnerable populations and cannot rely solely on wearing masks or personal protective equipment to fulfill their professional obligations to their patients. Also, healthcare facilities and their managers owe legal and ethical duties to their staff and patients for a safe healthcare environment. These facilities should consider a variety of protocols, educational interventions, and access options to increase COVID-19 vaccine uptake by their staff and patients. HCWs have an overwhelming need to become vaccinated and a moral obligation to serve as role models to encourage the public and their peers to engage in preventive health behaviors such as getting vaccinated with the available COVID-19 vaccines.

Several potential limitations should be considered regarding the results of this review. The search terms and methods used to identify relevant articles may have led to the omission of some relevant articles. Our criteria for selection (e.g., studies published in English during a certain time), may have introduced unknown biases. Uniformity in the primary outcome (declining to be vaccinated) differed significantly across studies (e.g., outright refusal, unlikely to take the vaccine, declined, disagreed with taking a vaccine, etc.) and may not be appropriately aggregated. Also, if a significant proportion of those who were unsure or undecided about COVID-19 vaccines end up declining the vaccine at a different period, the rates of hesitancy would be much higher than our estimates in this review. For the studies we included in this review, there were substantial differences in study participants based on age, gender, income, education, and professional roles (e.g., physicians versus nurses versus others). Aggregates presented in this review may not appropriately represent vaccine hesitancy for any particular group. Finally, this is a scoping review and not a quantitative 
meta-analysis. We cannot, therefore, comment on quantitative estimates such as the weighted estimates of vaccination hesitancy accounting for sample sizes and study variations.

\section{Conclusion}

This scoping review of healthcare workers' hesitancy to be COVID-19 vaccinated found that, on average, $22.51 \%$ out of 76,417 HCWs worldwide were hesitant about the available vaccines. Their main concerns (safety, efficacy, and side effects) were very similar to the concerns identified by the general public. Being male, older, and having more advanced education were enabling factors for the uptake of COVID-19 vaccination. Healthcare workers have a moral imperative and duty to play an important role in the acceptance of COVID19 vaccination by the public and their healthcare colleagues. Given the high rates of COVID-19 vaccination hesitancy in this population, education and policy-based interventions should be implemented to ensure that healthcare workers are vaccinated with the available COVID-19 vaccines. Without high compliance for COVID-19 vaccination among healthcare workers, there are enormous risks to the general public as well as healthcare workers.

Funding This research received no external funding.

\section{Declarations}

Conflict of interest Authors have no conflicts of interest to declare.

\section{References}

1. Anyiam-Osigwe, T (2021). COVID-19 vaccines are now approved in some countries. What will it take to approve them for the rest of the world? Retrieved from the GAVI website: https://www.gavi. org/vaccineswork/covid-19-vaccines-are-now-approved-somecountries-what-will-it-take-approve-them

2. Lazarus, J. V., Ratzan, S. C., Palayew, A., et al. (2021). A global survey of potential acceptance of a COVID-19 vaccine. Nature Medicine, 27(2), 225-228

3. Khubchandani, J., Sharma, S., Price, J. H., et al. (2021). COVID19 vaccination hesitancy in the United States: A rapid national assessment. Journal of Community Health, 46(2), 270-277

4. Sallam, M. (2021). COVID-19 Vaccine hesitancy worldwide: A concise systematic review of vaccine acceptance rates. Vaccines, 9(2), 160. https://doi.org/10.3390/vaccines9020160

5. Murphy, J., Vallières, F., Bentall, R. P., Shevlin, M., McBride, O., Hartman, T. K., McKay, R., Bennett, K., Mason, L., GibsonMiller, J., Levita, L., \& Hyland, P. (2021). Psychological characteristics associated with COVID-19 vaccine hesitancy and resistance in Ireland and the United Kingdom. Nature Communications, 12(1), 1-15

6. Courage, K.H (2021). It's essential to understand why some health care workers are putting off vaccination. Retrieved from https://www.vox.com/22214210/covid-vaccine-health-care-worke rs-safety-fears

7. Roy, B., Kumar, V., \& Venkatesh, A. (2020). Health care workers' reluctance to take the COVID-19 Vaccine: A consumer-marketing approach to identifying and overcoming hesitancy. NEJM Catalyst Innovations in Care Delivery. https://doi.org/10.1056/CAT. 20.0676

8. Hamel, L., et al.. (2020). KFF COVID-19 vaccine monitor: December 2020. Retrieved from https://www.kff.org/coronaviruscovid-19/report/kff-covid-19-vaccine-monitor-december-2020/

9. Gharpure, R., Guo, A., Bishnoi, C. K., Patel, U., Gifford, D., Tippins, A., Jaffe, A., Shulman, E., Stone, N., Mungai, E., \& Bagchi, S. (2021). Early COVID-19 first-dose vaccination coverage among residents and staff members of skilled nursing facilities participating in the pharmacy partnership for long-term care programUnited States, December 2020-January 2021. Morbidity and Mortality Weekly Report, 70(5), 178

10. Nguyen, K. H., Srivastav, A., Razzaghi, H., et al. (2021). COVID19 vaccination intent, perceptions, and reasons for not vaccinating among groups prioritized for early vaccination-United States, September and December 2020. Morbidity and Mortality Weekly Report., 70(6), 217

11. Papagiannis, D., Malli, F., Raptis, D. G., et al. (2020). Assessment of knowledge, attitudes, and practices towards new coronavirus (SARS-CoV-2) of health care professionals in Greece before the outbreak period. International Journal of Environmental Research and Public Health, 17(14), 4925

12. Wang, K., Wong, E. L. Y., Ho, K. F., et al. (2020). Intention of nurses to accept coronavirus disease 2019 vaccination and change of intention to accept seasonal influenza vaccination during the coronavirus disease 2019 pandemic: A cross-sectional survey. Vaccine, 38(45), 7049-7056

13. Dror, A. A., Eisenbach, N., Taiber, S., et al. (2020). Vaccine hesitancy: the next challenge in the fight against COVID-19. European Journal of Epidemiology, 35(8), 775-779

14. Fu, C., Wei, Z., Pei, S., Li, S., Sun, X., \& Liu, P. (2020). Acceptance and preference for COVID-19 vaccination in health-care workers (HCWs). MedRxiv. https://doi.org/10.1101/2020.04.09. 20060103

15. Kwok, K. O., Li, K. K., Wei, W. I., et al. (2021). Influenza vaccine uptake, COVID-19 vaccination intention and vaccine hesitancy among nurses. International Journal of Nursing Studies, 114, 103854

16. Nzaji, M. K., et al. (2020). Acceptability of vaccination against COVID-19 among healthcare workers in the Democratic Republic of the Congo. Pragmatic and Observational Research, 11, 103. https://doi.org/10.2147/POR.S271096

17. Detoc, M., Bruel, S., Frappe, P., Tardy, B., Botelho-Nevers, E., \& Gagneux-Brunon, A. (2020). Intention to participate in a COVID19 vaccine clinical trial and to get vaccinated against COVID-19 in France during the pandemic. Vaccine, 38(45), 7002-7006

18. Harapan, H., Wagner, A. L., Yufika, A., et al.. (2020). Acceptance of a COVID-19 vaccine in southeast Asia: A cross-sectional study in Indonesia. Frontiers in Public Health 8.

19. Gagneux-Brunon, A., Detoc, M., Bruel, S., Tardy, B., Rozaire, O., Frappe, P., \& Botelho-Nevers, E. (2021). Intention to get vaccinations against COVID-19 in French healthcare workers during the first pandemic wave: A cross-sectional survey. Journal of Hospital Infection, 108, 168-173

20. Schwarzinger, M., Watson, V., Arwidson, P., Alla, F., \& Luchini, S. (2021). COVID-19 vaccine hesitancy in a representative working-age population in France: A survey experiment based on vaccine characteristics. Lancet Public Health. https://doi.org/ 10.1016/S2468-2667(21)00012-8

21. Askarian, M., Fu, L., Taghrir, M. H., et al.. (2020). Factors affecting COVID-19 vaccination intent among Iranians: COVID-19 
vaccination acceptance. Retrieved from https://ssrn.com/abstr act $=3741968$

22. Ditekemena, J. D., Nkamba, D. M., Mavoko, A. M., et al. (2021). COVID-19 vaccine acceptance in the Democratic Republic of Congo: A cross-sectional survey. Vaccines, 9(2), 153

23. Grech, V., Bonnici, J., \& Zammit, D. (2020). Vaccine hesitancy in Maltese family physicians and their trainees vis-a-vis influenza and novel COVID-19 vaccination. Early Human Development. https://doi.org/10.1016/j.earlhumdev.2020.105259

24. Grech, V., Gauci, C., \& Agius, S. (2020). Vaccine hesitancy among Maltese healthcare workers toward influenza and novel COVID-19 vaccination. Early Human Development. https://doi. org/10.1016/j.earlhumdev.2020.105213

25. Kose, S., Mandiracioglu, A., Sahin, S., et al. (2020). Vaccine hesitancy of the COVID-19 by health care personnel. International Journal of Clinical Practice. https://doi.org/10.1111/ijcp.13917

26. Gadoth, A., Halbrook, M., Martin-Blais, R., et al. (2021). Crosssectional Assessment of COVID-19 vaccine acceptance among health care workers in Los Angeles. Annals of Internal Medicine. https://doi.org/10.7326/M20-7580

27. Barry, M., Temsah, M. H., Alhuzaimi, A., et al.. (2020). COVID19 vaccine confidence and hesitancy among healthcare workers: a cross-sectional survey from a MERS-CoV experienced nation. https://doi.org/10.1101/2020.12.09.20246447

28. Shekhar, R., Sheikh, A. B., Upadhyay, S., et al. (2021). COVID19 vaccine acceptance among health care workers in the United States. Vaccines, 9(2), 119

29. Verger, P., Scronias, D., Dauby, N., et al.. (2021). Attitudes of healthcare workers towards COVID-19 vaccination: Survey in France, French-speaking parts of Belgium and Canada Eurosurveillance, 26(3).

30. Eguia, H., Vinciarelli, F., Bosque-Prous, M., Kristensen, T., \& Saigí-Rubió, F. (2021). Spain's hesitation at the gates of a COVID-19 vaccine. Vaccines, 9(2), 170

31. Di Gennaro, F., Murri, R., Segala, F. V., et al. (2021). Attitudes towards Anti-SARS-CoV2 vaccination among healthcare workers: Results from a national survey in Italy. Viruses, 13(3), 371. https:// doi.org/10.3390/v13030371

32. Temsah, M. H., Barry, M., Aljamaan, F., et al. (2020). Adenovirus and RNA-based COVID-19 vaccines: Perceptions and acceptance among healthcare workers. medRxiv https://doi.org/10.1101/ 2020.12.22.20248657

33. Kuter, B. J., Browne, S., Momplaisir, F. M., et al. (2021). Perspectives on the receipt of a COVID-19 vaccine: A survey of employees in two large hospitals in Philadelphia. Vaccine. https:// doi.org/10.1016/j.vaccine.2021.02.029

34. Jain, V., Doernberg, S. B., Holubar, M., et al. (2021). Healthcare personnel knowledge, motivations, concerns and intentions regarding COVID-19 vaccines: A cross-sectional survey. medRxiv https://doi.org/10.1101/2021.02.19.21251993

35. Meyer, M. N., Gjorgjieva, T., \& Rosica, D. (2020). Healthcare worker intentions to receive a COVID-19 vaccine and reasons for hesitancy: A survey of 16,158 health system employees on the eve of vaccine distribution. medRxiv https://doi.org/10.1101/2020.12. 19.20248555

36. Shaw, J., Stewart, T., Anderson, K. B., et al. (2021). Assessment of US health care personnel (HCP) attitudes towards COVID-19 vaccination in a large university health care system. Clinical Infectious Diseases. https://doi.org/10.1093/cid/ciab054

37. Qattan, A., Alshareef, N., Alsharqi, O., Al Rahahleh, N., Chirwa, G. C., \& Al-Hanawi, M. K. (2021). Acceptability of a COVID-19 vaccine among healthcare workers in the Kingdom of Saudi Arabia. Frontiers in Medicine, 8, 83. https://doi.org/10.3389/fmed. 2021.644300

38. Papagiannis, D., Rachiotis, G., Malli, F., et al. (2021). Acceptability of COVID-19 vaccination among Greek health professionals. Vaccines, 9(3), 200

39. Ledda, C., Costantino, C., Cuccia, M., Maltezou, H. C., \& Rapisarda, V. (2021). Attitudes of Healthcare Personnel towards Vaccinations before and during the COVID-19 Pandemic. International Journal of Environmental Research and Public Health, 18(5), 2703

40. Desveaux, L., Savage, R. D., Tadrous, M., Kithulegoda, N., Thai, K., Stall, N. M., \& Ivers, N. M. (2021). Beliefs associated with intentions of non-physician healthcare workers to receive the COVID-19 vaccine in Ontario. Canada. https://doi.org/10.1101/ 2021.02.19.21251936

41. Mohamed-Hussein, A. A., Makhlouf, H., Abd El Aal, H., Kholief, K., Saad, M. M., \& Abdellal, D. A. (2021). A national survey of potential acceptance of COVID-19 vaccines in healthcare workers in Egypt. medRxiv. https://doi.org/10.1101/2021.01.11.21249324

42. Amin, D. P., \& Palter, J. S. (2021). COVID-19 vaccination hesitancy among healthcare personnel in the emergency department deserves continued attention. American Journal of Emergency Medicine. https://doi.org/10.1016/j.ajem.2021.01.089

43. Kociolek, L. K., Elhadary, J., Jhaveri, R., Patel, A. B., Stahulak, B., \& Cartland, J. (2021). Coronavirus disease 2019 vaccine hesitancy among children's hospital staff: A single-center survey. Infection Control \& Hospital Epidemiology. https://doi.org/10. 1017/ice.2021.58

44. Schrading, W. A., Trent, S. A., Paxton, J. H., et al. (2021). Vaccination rates and acceptance of SARS-CoV-2 vaccination among US emergency department health care personnel. Academic Emergency Medicine. https://doi.org/10.1111/acem.14236

45. Szmyd, B., Karuga, F. F., Bartoszek, A., et al. (2021). Attitude and behaviors towards SARS-CoV-2 vaccination among healthcare workers: A cross-sectional study from Poland. Vaccines., 9(3), 218. https://doi.org/10.3390/vaccines9030218

46. AP-NORC Center for Public Affairs Research (2021). Safety concerns remain the main driver of vaccine hesitancy. Retrieved from https://apnorc.org/projects/safety-concerns-remain-main-driverof-vaccine-hesitancy/

47. Udow-Phillips, M., \& Lantz, P. M. (2020). Trust in public health is essential amid the COVID-19 pandemic. Journal of Hospital Medicine, 15(7), 431-433

48. Gur-Arie, R., Jamrozik, E., \& Kingori, P. (2021). No jab, no job? Ethical issues in mandatory COVID-19 vaccination of healthcare personnel. BMJ Global Health, 6(2), e004877

Publisher's Note Springer Nature remains neutral with regard to jurisdictional claims in published maps and institutional affiliations. 